\title{
Linfadenite granulomatosa em suínos: linfonodos afetados e diagnóstico patológico da infecção causada por agentes do Complexo Mycobacterium avium ${ }^{1}$
}

\author{
Nelson Morés ${ }^{2 *}$, Lauren Ventura ${ }^{3}$, Valéria Dutra ${ }^{4}$, Virgínia S. Silva ${ }^{2}$, Waldomiro \\ Barioni Jr ${ }^{5}$, Salete R. Oliveira ${ }^{2}$, Beatris Kramer $^{2}$ e José S. Ferreira Neto ${ }^{6}$
}

\begin{abstract}
Morés N., Ventura L., Dutra V., Silva V.S., Barioni Jr W., Oliveira S.R, Kramer B. \& Ferreira Neto J.S. 2007. [Granulomatous lymphadenitis in swine: lymph nodes affected and pathologic diagnosis of the infection caused by Mycobacterium avium Complex agents.] Linfadenite granulomatosa em súnos: linfonodos afetados e diagnóstico patológico da infecção causada por agentes do Complexo Mycobacterium avium. Pesquisa Veterinária Brasileira 27(1):13-17. Embrapa Suínos e Aves, Cx. Postal 21, Concórdia, SC 89700-000, Brazil. E-mail: mores@cnpsa.embrapa.br

A study to evaluate the distribution of granulomatous lesions in the lymph nodes of swine carcasses was accomplished. The main objective was to compare the methods of isolation of mycobacteria of the Mycobacterium avium Complex (MAC), Ziehl-Neelsen (ZN) staining, histopathological examination and immunohistochemistry (IHC) for the diagnosis of granulomatous lymphadenitis caused by MAC. A total of 431 samples of lymph nodes from 394 swine carcasses kept in 12 cold storage rooms of southern Brazil, which had the Service of Federal Inspection (SFI), were analyzed. Lymph nodes with granulomatous lesions were submitted to histological examination, ZN staining and IHC with monoclonal antibody produced with cellular extract of M. avium. The agreement between the exams was measured by the Kappa test at a 95\% confidence level. The macrocospic examination done by SFI correctly identified $90.3 \%$ of granulomatous lesions, when compared with histological examination, and the majority of carcasses (92.5\%) showed the lesions only in lymph nodes of the alimentary chain. Histological examination confirmed the presence of granulomatous lesions in $90.3 \%$ of the lymph nodes. The agreements between histological examination, ZN staining (Kappa: 0.342), IHC and the isolation of MAC (Kappa: 0.102) were low, however high between IHC and histology with typical presence of granulomas in the lymph nodes (Kappa: 0.973). IHC together with histological examination revealed to be efficient for identification of the granulomatous lymphadenitis caused by MAC.
\end{abstract}

INDEX TERMS: Swine, granulomatous lymphadenitis, Mycobacterium avium, immunohistochemistry.

RESUMO.- Realizou-se um estudo com o objetivo de avaliar a distribuição de lesões granulomatosas nos linfonodos das carcaças de suínos abatidos e de comparar os métodos de isolamento do Complexo Mycobacterium avium (MAC), coloração de

\footnotetext{
${ }^{1}$ Recebido em 18 de agosto de 2006.

Aceito para publicação em 8 de setembro de 2006.

2 Embrapa Suínos e Aves, BR 153 Km 110, Cx. Postal 21, Concórdia, SC 89700-000. *Autor para correspondência: mores@cnpsa.embrapa.br

${ }^{3}$ CEDISA, BR 153, Km 110, Cx. Postal 273, Concórdia, SC 89700-000.

${ }^{4}$ Universidade Federal de Mato Grosso, Av. Fernando Corrêa da Costa s/n, Bairro Coxipó, Cuiabá, MT 78060-900.

${ }^{5}$ Embrapa Instrumentação Agropecuária, Rua XV de Novembro 1452, São Carlos, SP 13560-970.

${ }^{6}$ Depto Medicina Veterinária Preventiva e Saúde Animal, FMVZ-USP, Av. Professor Dr. Orlando Marques de Paiva, Cidade Universitária, São Paulo, SP 05508-270.
}

Ziehl-Neelsen (ZN), exames histopatológico e de imunohistoquímica (IHQ) para o diagnóstico da linfadenite granulomatosa causada por micobactérias do MAC. Foram utilizadas 431 amostras de linfonodos colhidos de 394 carcaças de suínos abatidos em 12 frigoríficos da Região Sul do Brasil, com o Serviço de Inspeção Federal (SIF). Os linfonodos que apresentavam lesões granulomatosas foram submetidos aos exames histológicos, ZN e IHQ com anticorpo monoclonal produzido com extrato celular de M. avium. A concordância entre os exames foi medida pelo teste Kappa, com nível de confiança de 95\%. O exame macroscópico realizado pelo SIF identifica corretamente $90,3 \%$ das lesões granulomatosas, quando comparado com o exame histológico e a maioria das carcaças $(92,5)$ apresentam lesões apenas nos linfonodos cadeia alimentar. $\mathrm{O}$ exame histológico confirmou a presença de lesões granulomatosas em 90,3\% dos linfonodos. As concordâncias entre os 
exames histopatológico e coloração de ZN (Kappa: 0,342) e de IHQ e o isolamento do MAC (Kappa: 0,102) foram baixas, porém alta entre os exames de IHQ e histológico com a presença de granulomas típicos nos linfonodos (Kappa: 0,973). O exame de IHQ associado ao exame histopatológico mostrou-se eficiente na identificação das lesões de linfadenite granulomatosa causadas pelo MAC.

TERMOS DE INDEXAÇÃO: Suínos, linfadenite granulomatosa, Mycobacterium avium, imunohistoquímica.

\section{INTRODUÇÃO}

A linfadenite granulomatosa em suínos, causada por micobac-térias do Complexo Mycobacterium avium (MAC), é uma doença que não altera o desempenho dos animais, mas é de importância econômica devido à condenação ou destino condicionado das carcaças afetadas. Seu impacto econômico para a suinocultura da Região Sul do Brasil foi estimado em 5,8, 7,0 e 8,0 milhões de reais em 1997, 1998 e 1999, respectivamente (Martins 2001). Em rebanhos suínos, o diagnóstico presuntivo da infecção pelo MAC in vivo pode ser feito com o teste de tuberculina pareada, utilizando-se PPD aviária e mamífera (Morés et al. 2006), o que permite distinguir tuberculose clássica por Mycobacterium bovis das infecções causadas pelo MAC. Entretanto, há dificuldades para o diagnóstico etiológico por isolamento do agente; pois isso exige procedimentos especiais de laboratórios, demanda muito tempo, tem baixa sensibilidade e custo elevado de execução (Thoen 1992). As lesões observadas nos linfonodos são do tipo granulomatosas (Balian et al. 1995), mas também não são conclusivas, uma vez que podem ser provocadas por outras bactérias, como Mycobacterium bovis e Rhodococcus equi (Thoen 1992, Silva et al. 2001). A imunohisto-química (IHQ), associada ao exame histopatológico, poderá fornecer um diagnóstico etiológico seguro e rápido, favorecendo a tomada de decisão para seu controle.

O objetivo deste trabalho foi estudar a distribuição das lesões granulomatosas nos linfonodos das carcaças e comparar técnicas de identificação do agente em cortes histológicos de tecidos incluídos em parafina, para diagnóstico da linfadenite granulomatosa causada pelo MAC.

\section{MATERIAL E MÉTODOS}

Foram utilizadas 394 carcaças de suínos abatidos em 12 frigoríficos que tinham Serviço de Inspeção Federal (SIF), localizados nos Estados do Paraná, Santa Catarina e Rio Grande do Sul. Esses suínos provinham de granjas com histórico de condenações de carcaças por linfadenite granulomatosa. Para cada carcaça afetada anotaram-se os linfonodos que apresentavam lesões granulomatosas, e fragmentos destes foram colhidos em formol a $10 \%$ tamponado e processados para exame histológico de rotina (Luna 1968), e outros fragmentos foram acondicionados sob refrigeração para isolamento microbiológico, cujos resultados já foram publicados (Silva et al. 1998). Lâminas histológicas foram usadas para pesquisa de bacilos álcoolácido-resistentes (BAAR), pela coloração de Ziehl-Neelsen (Luna 1968). Para exame de imunohistoquímica (IHQ) foram utilizados linfonodos com lesões granulomatosas de 216 suínos apreendidos pelo SIF e selecionados das 394 carcaças descritas anteriormente, dos quais 112 eram positivos e 104 negativos para o Complexo Mycobacterium avium (MAC) no exame microbiológico (Silva et al. 2001). Os linfonodos foram fixados em formalina $10 \%$ tamponada e processados conforme rotina para obtenção de cortes histológicos de 5 micra. No exame de
IHQ para coloração das lâminas, utilizou-se a técnica do complexo Avidina-Biotina-Peroxidase (Vectastainâ Kit ABC). Como anticorpo primário foi utilizado um anticorpo monoclonal (IgG1) produzido com extrato celular de Mycobacterium avium (QED Bioscience Inc. Cat. 18104). Para revelação da reação, utilizou-se o substrato $3,3-$ diaminobenzidine (DAB) e, como coloração de fundo, a hematoxilina de Harris. Em cada bateria de exame foram incluídas uma lâmina controle positiva obtida de suínos inoculados com micobactérias do MAC, uma lâmina negativa obtida de suíno SPF (Specificy Pathogens Free) para micobactérias e, para cada lâmina testada, incluiu-se uma lâmina do mesmo bloco de tecido, substituindo-se o anticorpo monoclonal 18104 por PBS. Para interpretação da reação, considerou-se a quantidade de antígeno com reação positiva na lâmina: reação negativa, + reação positiva com pouca quantidade de antígeno, ++ positiva com quantidade moderada de antígeno, +++ reação positiva acentuada presença de antígeno. Para controle de especificidade para o anticorpo 18104 foram usados os fígados de cinco hamsters inoculados com Mycobacterium bovis, com lesões granulomatosas. A concordância entre os exames histopatológico e de Ziehl-Neelsen, entre o isolamento do MAC e a IHQ, e entre a IHQ e exame histológico foi medida pelo teste Kappa, com nível de confiança de 95\% (WIN Episcope 2.0 2004)

\section{RESULTADOS E DISCUSSÃO}

O Quadro 1 apresenta os resultados dos linfonodos afetados, no exame macroscópico realizado pelo SIF, compatíveis com linfadenite granulomatosa (Fig.1).

Os linfonodos da cadeia alimentar (cefálicos e/ou mesentéricos) foram os mais afetados, ocorrendo em 95,2\% das carcaças. Em 4,5\% das carcaças observaram-se lesões, ao mesmo tempo, nos linfonodos da cadeia alimentar e nos mediastínicos. Em apenas uma carcaça os linfonodos cefálicos e/ou mesentéricos não estavam envolvidos, onde se encontraram lesões isoladamente nos linfonodos inguinais. A predominância de lesões nos linfonodos cefálicos e/ou mesentérico nas infecções pelo MAC é fato observado por outros autores (Ferreira Neto et al. 1989, Balian et al. 1995, Pavlik et al. 2003, Matlova et al. 2004).

Das 394 carcaças apreendidas pelo SIF, devido à presença de linfadenite granulomatosa, foram examinados 431 linfonodos pela histologia de rotina e a presença de BAAR (Quadro 2). Destes, confirmou-se a presença de lesões granulomatosas em 389 deles $(90,3 \%)$, indicando boa confiabilidade do exame macroscó-pico realizado na inspeção das vísceras, concordando com os achados de Ferreira Neto et al. (1989). Os granulomas eram constituídos de macrófagos diferenciados em células epitelióides, arranjados concentricamen-

\section{Quadro 1. Freqüência de linfonodos com lesões de linfadenite granulomatosa em 394 carcaças afetadas e seus respectivos destinos (avaliação macroscópica feita pelo médico veterinário do SIF)}

\begin{tabular}{lc}
\hline \multicolumn{1}{c}{ Linfonodos afetados } & Frequiência (\%) \\
\hline Mesentéricos & $267(67,8)$ \\
Cefálicos & $24(6,1)$ \\
Mesentéricos + cefálicos & $84(21,3)$ \\
Mesentéricos + cefálicos + mediastínicos (Me) & $4(1,0)$ \\
Mediastinicos & $0(0,0)$ \\
Mediastinicos + cefálicos & $4(1,0)$ \\
Mesentéricos + mediastínicos & $10(2,5)$ \\
Inguinais & $1(0,3)$ \\
Totais & $394(100,0)$
\end{tabular}




\section{Quadro 2. Concordância entre os exames histopatológicos pela coloração de hematoxilina e eosina e a presença de BAAR na coloração de Ziehl-Neelsen (ZN) de 431 linfonodos classificados como linfadenite granulomatosa pelo SIF na linha de abate, oriundos de 394 carcaças $^{\mathrm{a}}$}

\begin{tabular}{lccc}
\hline Exame histopatológico & Frequiência & \multicolumn{2}{c}{ Exame de coloração de ZN \%) } \\
\cline { 3 - 4 } & $(\%)$ & Positivo & Negativo \\
\hline Lesão granulomatosa & $389(90,3)$ & $283(72,8)$ & $106(27,2)$ \\
Outro tipo de lesão & $38(8,8)$ & 0 & $38(100,0)$ \\
Sem lesão & $4(0,9)$ & 0 & $4(100,0)$ \\
Total & $431(100,0)$ & $283(65,7)$ & $148(34,3)$
\end{tabular}

a Concordância global observada entre os exames: 0,754; Kappa: 0,342 \pm 0,036 .

te com ou sem áreas centrais necróticas caseificadas e/ou calcificadas e com proliferação de tecido fibroso (Fig.2), às vezes formando uma cápsula externa (Fig.3), conforme já descrito (Senk et al. 1992, Balian et al. 1995). Houve concordância de $75,4 \%$ entre o exame histopatológico e a presença de BAAR nas lesões. Margolis et al.

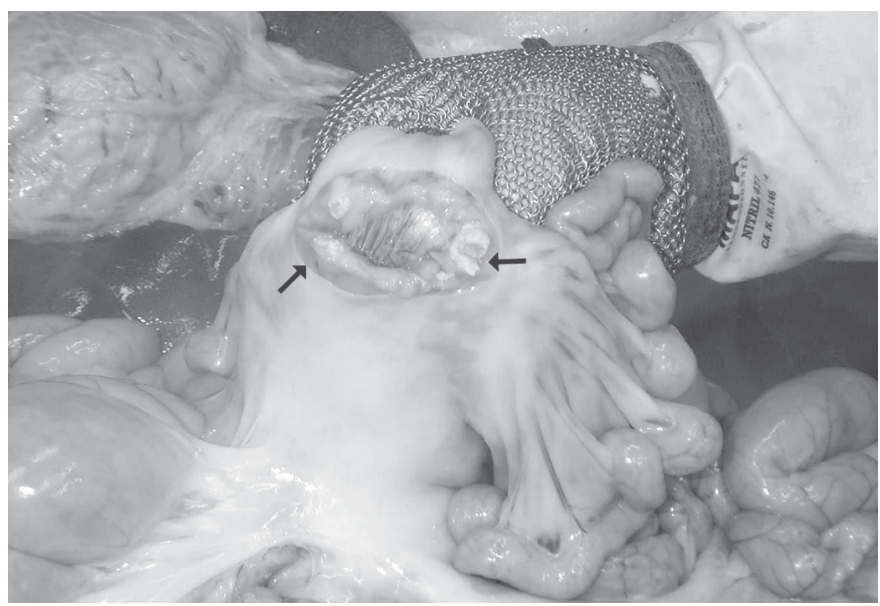

Fig.1. Granuloma em linfonodo mesentérico de suíno (setas) infectado com micobactérias do Complexo Mycobacterium avium (MAC), com lesão.

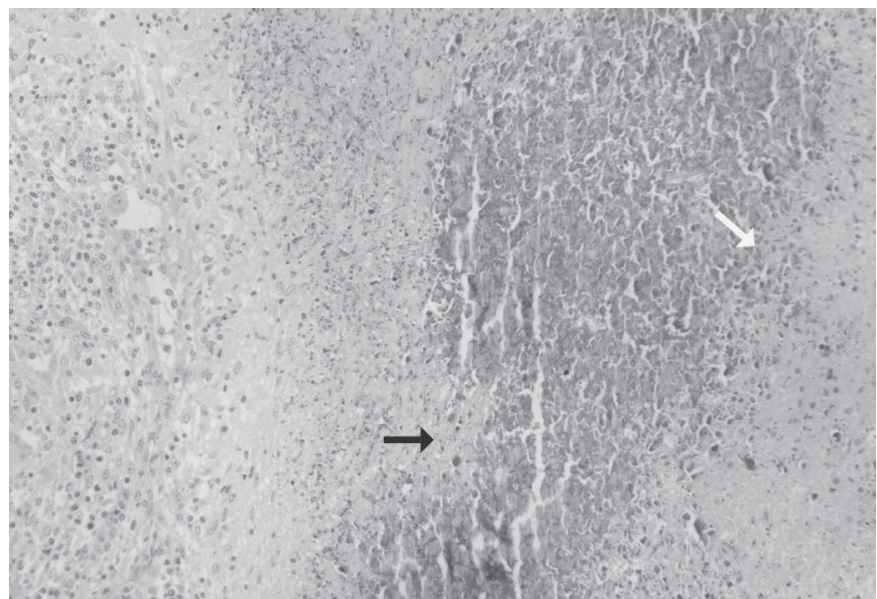

Fig.2. Necrose de caseificação com mineralização (seta branca) e início de proliferação conjuntiva (seta preta), em granuloma de linfonodo de suíno infectado pelo MAC. HE, obj.25.

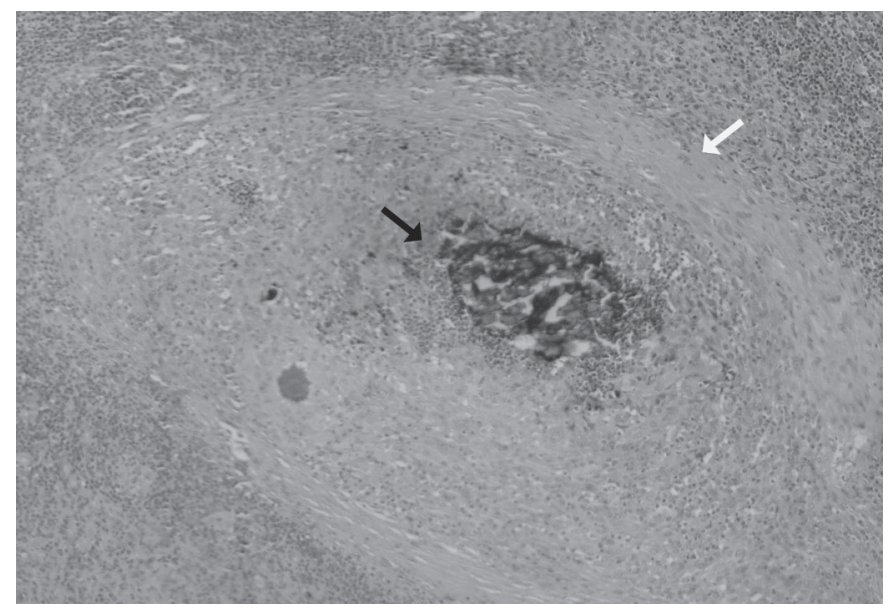

Fig.3. Granuloma com cápsula fibrosa (seta branca) e necrose de caseificação e mineralização (seta preta), em linfonodo de suíno infectado pelo MAC. HE, obj.25.

(1994) encontraram sensibilidade de $86 \%$ para o exame histopatológico e apenas de $15 \%$ para a coloração ácida, considerando o isolamento de micobactérias como teste padrão. Neste trabalho, foi possível encontrar BAAR em 72,8\% dos linfonodos com lesões granuloma-tosas no exame histológico.

No Quadro 3, estão os resultados de IHQ dos linfonodos lesados, oriundos de suínos com e sem isolamento do MAC. Dos 112 suínos em que micobactérias do MAC foram isoladas, $93\left(83,0^{\circ}\right)$ foram positivos e $19(17,0 \%)$ negativos no teste de IHQ. Entretanto, os 93 suínos, cujos linfonodos apresentaram lesões caracterizadas por granulomas típicos com necrose de caseifi-cação e/ou mineralização (Senk et al. 1992), todos foram positivos no teste de IHQ e os 19 que não tinham granuloma típico de infecção por micobactérias, todos foram negativos. Por outro lado, dos 104 suínos em que o MAC não foi isolado, $76(73,1 \%)$ foram positivos e $28(26,9 \%)$ negativos no teste IHQ, e dos 76 suínos que foram positivos no teste de IHQ, apenas dois $(2,6 \%)$ não apresentavam lesões granulomatosas típicas da infecção por micobactérias (Senk et al. 1992). Quando foram comparados os exames de IHQ com o de isolamento do MAC, houve fraca concordância entre os testes (Kappa igual a 0,102 $\pm 0,057$ ) (Quadro 4). Entretanto, houve forte concordância (Quadro 5) entre os exames de IHQ em corte de tecido e a presença de lesões histopatológicas caracterizadas pela presença de granulomas típicos nos linfonodos (Kappa igual a 0,973 $\pm 0,068$ ). A reação positiva no teste de IHQ estava localizada na massa necrótica no interior dos granulomas, principalmente no citoplasma de macrófagos degenerados, localizados na periferia das áreas de necrose e/ou mineralização, e que apresentavam diferentes quantidades de antígenos marcados (Fig.4-6). Isso sugere que $o$ anticorpo utilizado identifica antígenos de secreção do MAC, que são fagocitados pelos macrófagos. Os resultados indicam que o exame histopatológico, com presença de gra-nuloma típico e necrose e/ou mineralização da massa necrótica, seguido do teste de IHQ com o anticorpo monoclonal 18104, foi o procedimento mais adequado para o diagnóstico da linfadenite pelo MAC. Os resultados do teste de IHQ nos tecidos de hamsters foram todos negativos, indicando que o anticorpo monoclonal não reconhece antígenos de Mycobacterium bovis no método utilizado.

Lesões macroscópicas semelhantes à tuberculose freqüientemente são encontradas, pelo serviço de inspeção sanitária de 
Quadro 3. Exame imunohistoquímico (IHQ) em linfonodos de suínos com lesão granulomatosa no abate, com e sem isolamento de micobactérias do Complexo Mycobacterium avium (MAC)

\begin{tabular}{|c|c|c|c|c|c|c|c|}
\hline \multirow{2}{*}{$\begin{array}{c}\text { Isolamento } \\
\text { de MAC }\end{array}$} & \multicolumn{2}{|c|}{ Resultados do exame de IHQ } & \multicolumn{4}{|c|}{ Tipos de alterações histopatológicas ${ }^{a}$} & \multirow[t]{2}{*}{ Totais } \\
\hline & & & a & b & c & $\mathrm{d}$ & \\
\hline \multirow[t]{5}{*}{ Positivo } & Negativo $(\mathrm{n}=19)$ & b & 0 & 5 & 1 & 13 & 19 \\
\hline & & + & 16 & 0 & 0 & 0 & 16 \\
\hline & Positivo ( $\mathrm{n}=93$ ) & ++ & 20 & 0 & 0 & 0 & 20 \\
\hline & & +++ & 37 & 0 & 0 & 0 & 37 \\
\hline & Totais & & 93 & 5 & 1 & 13 & 112 \\
\hline \multirow[t]{5}{*}{ Negativo } & Negativo $(\mathrm{n}=28)$ & - & 0 & 1 & 15 & 12 & 28 \\
\hline & & + & 27 & 0 & 0 & 2 & 29 \\
\hline & Positivo $(\mathrm{n}=76)$ & ++ & 17 & 0 & 0 & 0 & 17 \\
\hline & & +++ & 30 & 0 & 0 & 0 & 30 \\
\hline & Totais & & 74 & 1 & 15 & 14 & 104 \\
\hline
\end{tabular}

\footnotetext{
a a = Granuloma típico com necrose de caseificação e mineralização, b = Granuloma com presença ou ausência de células gigantes, mas sem necrose de caseificação e mineralização, $\mathrm{c}=$ Presença de vesículas com delgada cápsula conjuntiva e/ou apenas fibrose, $\mathrm{d}=$ Linfonodo sem lesão no exame histopatológico.

b - Reação ausente, + positiva fraca, ++ positiva evidente, +++ positiva.
}

Quadro 4. Resultados comparativos do exame imunohistoquímico (IHQ) e de isolamento de micobactérias do Complexo Mycobacterium avium (MAC) de suínos de abate com lesão macroscópoica de linfadenite granulomatosa ${ }^{a}$

\begin{tabular}{ccccc}
\hline \multirow{2}{*}{ Exames } & \multicolumn{2}{c}{ Exame IHQ } & \multirow{2}{*}{ Totais $(\%)$} \\
\cline { 2 - 4 } & & $+(\%)$ & $-(\%)$ & \\
\hline \multirow{2}{*}{ Isolamento de MAC } & + & $93(43,05)$ & $19(8,80)$ & $112(51,85)$ \\
Totais & - & $76(35,18)$ & $28(12,96)$ & $104(48,15)$ \\
& & $169(78,24)$ & $47(21,76)$ & $216(100,00)$
\end{tabular}

a Concordância global observada: 0,560; Kappa: 0,102 $\pm 0,057$.

Quadro 5. Resultados comparativos entre o exame histopatológico e imunohistoquímico (IHQ) para identificação da infecção por MAC em suínos de abate com lesão macroscópica de linfadenite granulomatosa ${ }^{a}$

\begin{tabular}{ccccc}
\hline \multirow{2}{*}{ Exames } & \multicolumn{2}{c}{ Exame de imunoperoxidase } & \multirow{2}{*}{ Totais (\%) } \\
\cline { 2 - 3 } & & $+(\%)$ & $-(\%)$ & \\
\hline \multirow{2}{*}{ Exame histopatológico $^{\mathrm{b}}$} & + & $167(77,31)$ & $0(0,00)$ & $167(77,31)$ \\
& - & $2(0,93)$ & $47(21,76)$ & $49(22,69)$ \\
Totais & & $169(78,24)$ & $47(21,76)$ & $216(100,00)$
\end{tabular}

a Concordância global observada: 0,991; Kappa: 0,973 $\pm 0,068$.

b Granuloma típico com necrose de caseificação e mineralização.

carnes, em linfonodos do trato digestivo de suínos oriundos de granjas modernas. Dessas lesões têm sido isolados, predominantemente, microorganismos pertencentes ao MAC (Szabó et al. 1975, Silva et al. 2001, Pavlik et al. 2003, Matlova et al. 2004). As lesões macro e microscópicas, encontradas no presente estudo, não são diferentes daquelas provocadas por Mycobacterium bovis e M. tuberculosis (Thoen 1992), não sendo, portanto, o exame histopatológico suficiente para identificar as espécies de micobactérias e estabelecer relação entre o tipo de micobactéria envolvida e as características das alterações histopatológicas (Ferreira Neto 1989, Thoen 1992). As lesões observadas em suínos infectados naturalmente, a exemplo daquelas encontradas no presente trabalho, são idênticas àquelas provo-cadas em suínos inoculados com o MAC (Senk et al. 1992, Silva 1998). Segun-

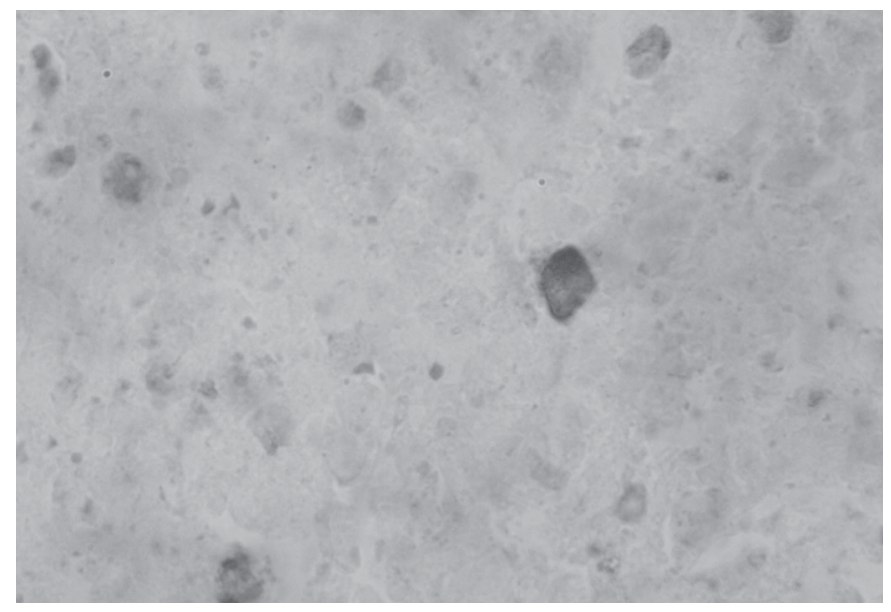

Fig.4. Restos celulares com reação positiva (+) para Mycobacterium avium na imunohistoquímica, em granuloma de linfonodo de suíno. Técnica da Avidina-Biotina-Peroxidase, obj.100.

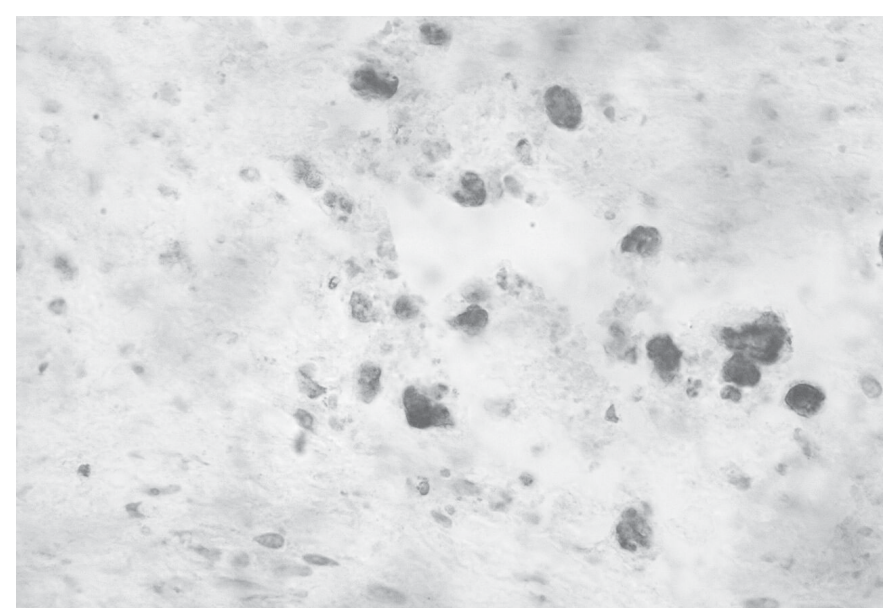

Fig.5. Restos celulares com reação positiva $(++)$ para Mycobacterium avium na imunihistoquímica, em granuloma de linfonodo de suíno. Técnica da Avidina-Biotina-Peroxidase, obj.100. 


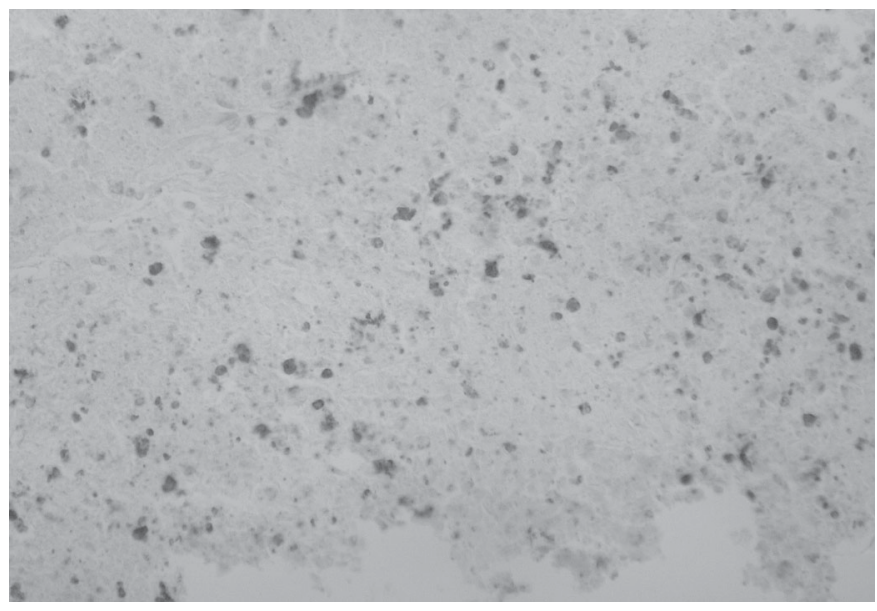

Fig.6. Restos celulares com reação positiva $(+++)$ para Mycobacterium avium na imunohisoquímica, em granuloma de linfonodo de suíno. Técnica da Avidina-Biotina-Peroxidase, obj.50

do Szabó et al. (1975), os dados epizootiológicos podem fornecer informações mais conclusivas sobre a etiologia das lesões granulomatosas encontradas nos linfonodos de suínos na linha de abate, do que apenas o exame histológico. Nos rebanhos, o teste de tuberculinização pareada (Morés et al. 2006) pode fornecer informação importante sobre o grupo de micobactérias envolvidas. Ademais, a presença de lesões granulomatosas limitadas aos linfonodos da cadeia digestiva, a origem dos suínos de uma gran-ja fechada, sem contato com outros mamíferos, e a ausência de produtos lácteos na dieta são sugestivos de infecção pelo MAC.

A localização das lesões nos tecidos dos suínos afetados é importante e define o destino das carcaças nos matadouros. Neste trabalho, os linfonodos da cadeia digestiva (mesentéricos e cefálicos) foram os mais afetados. A ocorrência exclusiva de lesões nesses linfonodos sugere fortemente o envolvimento de micobactérias do complexo MAC (Ferreira Neto et al. 1989, Balian et al. 1995, Pavlik et al. 2003, Matlova et al. 2004), demonstrado nesse estudo pelo teste de IHQ; em 18 carcaças foram encontradas lesões nos linfonodos mediastínicos, em associações com lesões nos linfonodos cefálicos e mesentéricos, mas sem envolvimento do pulmão e de linfonodos do aparelho respiratório. É provável que a origem das lesões nos linfonodos mediastínicos dessas carcaças tenha sido via digestiva, através do esôfago, e não via respiratória, pois a porção torácica do esôfago é drenada pelos linfonodos mediastínicos (Clair L.S. St 1967). Isto difere da infecção por $M$. bovis que, além de afetar órgãos parenquimatosos, como os pulmões, usa, como principal via de infecção, a respiratória (Thoen 1992).

\section{CONCLUSÕES}

O exame macroscópico feito pelo Serviço de Inspeção Federal (SIF) nos matadouros onde esse trabalho foi realizado, identificou corretamente $90,3 \%$ das lesões granulomatosas nos linfo-nodos, quando comparado com o exame histológico. A linfa-denite granulomatosa causada por bactérias do Complexo Mycobacterium avium (MAC) atinge predominantemente os linfonodos mesentéricos e/ou cefálicos. O exame de Imuno-histoquímica (IHQ) das lesões de linfadenite granulomatosa mostrou-se eficiente na identificação das infecções causadas pelo MAC, constituindo-se num teste seguro, rápido e econômico para o diagnóstico das alterações patológicas.

Agradecimentos.- Aos veterinários do Serviço de Inspeção Federal dos 12 abatedouros onde o trabalho foi realizado, pelo auxílio no exame macroscópico das carcaças e coleta dos linfonodos para exames laboratoriais. $\mathrm{O}$ Projeto foi financiado pelo Sindicato Catarinense de Carnes e Derivados de Santa Catarina, pela Embrapa e pela FAPESP.

\section{REFERÊNCIAS}

Balian S.C. 1995. Estudo de linfadenites tuberculóides em suínos abatidos em matadouro da região da Grande São Paulo, no período de 19931994: aspectos macroscópicos, histopatológicos e pesquisa de micobactérias. Dissertação de Mestrado em Epidemiologia Experimental e Aplicada a Zoonoses, Faculdade de Medicina Veterinária e Zootecnia, USP, São Paulo, SP. 76p.

Cair L.E.St 1967. Anatomia, p.3-37. In: Dunne H.W. (ed.) Enfermidades del Cerdo. $2^{\text {a }}$ ed. Union Topografica Editorial Hispano-Americana, Mexico.

Ferreira Neto J.S., Côrtes J.A., Sinhorini I.L., Vasconcellos S.A., Ito F.H. \& Silva E.A.M. 1989. A lesão tuberculóide macroscópica como critério dignóstico da infecção micobacteriana em suínos abatidos em matadouro. Revta Fac. Med. Vet. Zootec. USP 26(1):21-33.

Luna L.G. 1968. Manual of Histologic Staining of the Armed Forces Institute of Pathology. 3rd ed. McGraw-Hill, New York, p.258.

Margolis M.J., Hutchinson L.J., Kephart K.B., Hattel A.L., Whitloch R.H. \& Payeur J.B. 1994. Results of using histologic examination and acidfast staining to confirm a diagnosis of swine mycobacteriosis made on the basis of gross examination. J. Am. Vet. Med. Assoc. 204(10):15711572 .

Martins L.S. 2001. Epidemiologia e controle das micobacterioses em suínos no sul do Brasil: Estimativa do impacto econômico e estudo da sazonalidade. Dissertação de Mestrado em Epidemiologia Experimental, Faculdade de Medicina Veterinária e Zootecnia, USP, São Paulo, SP. $51 \mathrm{p}$.

Matlova L., Dvorska L., Palecek K., Maurenc L., Bartos M. \& Pavlik I. 2004. Impact of sawdust and wood shavings in bedding on pig tuberculous lesions in lymph nodes, and IS1245 FRLP analysis of Mycobacterim avium subsp. Hominissuis of serotypes 6 and 8 isolated from pigs and enviroment. Vet. Microbiol 102:227-236.

Morés N., Amaral A.L. Ventura L., Silva R.A.M. Silva V.S. \& Barioni Jr W. 2006. Estudo comparativo de métodos de tuberculinização, com tuberculina aviária e bovina, no diagnóstico da infecção por agentes do Complexo Mycobacterium avium ou M. bovis em suínos. Arq. Bras. Med. Vet. Zootec. (Em publicação)

Pavlik I., Matlova L., Dvorska L., Bartl J., Oktabcova L., Docekal J. \& Parmova I. 2003. Tuberculous lesions in pigs in the Czech Republic during 19901999: occurrence, causal factors and economic losses. Vet. Med. Czech 48(5):113-125.

Senk L., Pogacnilk M. \& Boehm O. 1992. Pathology of experimental Mycobacterium intracellulare serovar 4 infection in the pig. Proc. 12th Int. Pig Vet. Soc. Congress (IPVS), The Hague, p.342.

Silva V.S. 1998. Estudo da transmissão horizontal de Mycobacterium aviumintracellulare em suínos. Dissertação de Mestrado em Sanidade Animal, Faculdade de Veterinária, UFPel, Pelotas, RS. 47p.

Silva V.S., Morés N., Ferreira F., Dias R.A., Balian S., Dutra V., Leão S.C., Pinheiro S.R., Sakamoto S.M. \& Ferreira Neto J.S. 2001. Identificação dos fatores de risco associados a ocorrência de micobacterioses no Sul do Brasil, estudo caso-controle. Arqs Inst. Biológico, São Paulo, 68(2):19-22.

Szabó I., Tuboly S., Széky A., Kerekes J., Udvardy N. 1975. Swine lymphadenitis due to Mycobacterium avium and atypical mycobacteria. Acta Vet. Acad. Scient. Hung. 25:77-83.

Thoen C.O. 1992. Tuberculosis, p.617-26. In: Leman A.D., Straw B.E., Mengeling, W.L., Dallaire S. \& Taylor D.J. (ed.), Diseases of Swine. Iowa State University Press, Ames.

WIN Episcope 2.0. 2004. Disponível em: <http:www.clive.ed.ac.uk/winepiscope> . Acesso em: 18 agosto de 2004 